\title{
Implications of PCR and ELISA results on the routes of bulk-tank contamination with Mycobacterium avium ssp. paratuberculosis
}

\author{
A. Beaver, ${ }^{*} \dagger^{1}$ C. L. Cazer, $†$ P. L. Ruegg, $\ddagger$ Y. T. Gröhn, $\dagger$ and Y. H. Schukken $† \S$ \\ *Department of Animal Science, \\ †Department of Population Medicine and Diagnostic Sciences, College of Veterinary Medicine, and \\ ‡Department of Dairy Science, University of Wisconsin, Madison 53706 \\ §GD Animal Health Service, Deventer 7400 AA, the Netherlands
}

\section{ABSTRACT}

Mycobacterium avium ssp. paratuberculosis (MAP), the etiologic agent of Johne's disease in dairy cattle, may enter the bulk tank via environmental contamination or direct excretion into milk. Traditionally, diagnostics to identify MAP in milk target either MAP antibodies (by ELISA) or the organism itself (by culture or PCR). High ELISA titers may be directly associated with excretion of MAP into milk but only indirectly linked to environmental contamination of the bulk tank. Patterns of bulk-milk ELISA and bulk-milk PCR results could therefore provide insight into the routes of contamination and level of infection or environmental burden. Coupled with questionnaire responses pertaining to management, the results of these diagnostic tests could reveal correlations with herd characteristics or onfarm practices that distinguish herds with high and low environmental bulk-tank MAP contamination. A questionnaire on hygiene, management, and Johne's specific parameters was administered to 292 dairy farms in New York, Oregon, and Wisconsin. Bulk-tank samples were collected from each farm for evaluation by real-time PCR and ELISA. Before DNA extraction and testing of the unknown samples, bulk-milk template preparation was optimized with respect to parameters such as MAP fractionation patterns and lysis. Two regression models were developed to explore the relationships among bulk-tank PCR, ELISA, environmental predictors, and herd characteristics. First, ELISA optical density (OD) was designated as the outcome in a linear regression model. Second, the log odds of being PCR positive in the bulk tank were modeled using binary logistic regression with penalized maximum likelihood. The proportion of PCR-positive bulk tanks was highest for New York and for organic farms, providing a clue as to the geographical patterns of MAP-positive bulk-tank samples and relationship to production type. Bulk-milk

Received May 23, 2015.

Accepted October 17, 2015

${ }^{1}$ Corresponding author: ab2368@cornell.edu
PCR positivity was also higher for large relative to small herds. The models revealed that bulk-milk PCR result could predict ELISA OD, with PCR-positive results corresponding to high bulk-milk ELISA titers. Similarly, ELISA was a predictor of PCR result, although the association was stronger for organic farms. Despite agreement between high bulk-milk ELISA titers and positive PCR results, a large proportion of high ELISA farms had PCR-negative bulk tanks, suggesting that farms are able to maintain satisfactory hygiene and management despite a presence of MAP in these herds. Key words: Mycobacterium avium ssp. paratuberculosis, Johne's disease, bulk-tank milk, PCR, ELISA

\section{INTRODUCTION}

Mycobacterium avium ssp. paratuberculosis (MAP) is the causative agent of Johne's disease, a granulomatous enteritis that is both chronic and progressive; MAP affects predominantly ruminants and has been cultured from the milk and feces of both clinically infected and asymptomatic animals. Because MAP-positive animals may fail to manifest clinical signs, testing at the herd level remains an important tool to evaluate on-farm MAP presence and prevalence (Manning and Collins, 2001).

According to a study conducted by the National Animal Health Monitoring System (Lombard et al., 2013), the proportion of US dairy herds with MAP in their onfarm environments exceeds $70 \%$. This high prevalence, coupled with MAP's ability to survive common pasteurization practices (Sung and Collins, 1998; Grant et al., 2002, 2005), necessitates the optimization of simple, rapid diagnostic tests to determine herd MAP status and progression of MAP infection at the herd level. Current herd-level testing strategies commonly involve pooling fecal samples throughout the farm environment or from a large number of animals for evaluation by culture or PCR (Collins, 2011). Several of these strategies present certain drawbacks; for example, individual animal sampling may not be labor efficient and the fecal culture method is not timely, with results typically 
reported after 8 to $16 \mathrm{wk}$ of incubation. Optimization of bulk-tank milk testing would facilitate timely response to MAP contamination through alteration of critical on-farm management and hygiene practices (Cazer et al., 2013).

Viable MAP identified by culture methods has been found in both raw and pasteurized pooled milk fed to calves (Ruzante et al., 2008), thus conceivably perpetuating the cycle of infection. Additionally, milk and other dairy products may represent a route of transmission of MAP from cattle to humans. This transmission is particularly concerning because MAP has been implicated in the development of Crohn's disease in humans. Although MAP has not been definitively identified as a cause of Crohn's disease (Over et al., 2011), investigation of its potential as a zoonotic organism is of sufficient public health concern to justify the monitoring of bulk milk intended for consumers (Eltholth et al., 2009).

An "acceptable" threshold for MAP concentration in the bulk tank is unknown, mainly due to an absence of quantitative information on the likelihood of human disease as a consequence of MAP exposure (Weber et al., 2008) and the lack of confidence in pasteurization as a safeguarding tool to remove all viable MAP. Mycobacterium avium ssp. paratuberculosis bacteria in concentrations exceeding $10^{4}$ cells/L have been documented to survive HTST pasteurization (Grant et al., 2005). Thus, Weber et al. (2008) defined low-risk farms as those with a certain probability (approaching 100\% in simulated test schemes) of having a concentration $<10^{3}$ MAP organisms/L of milk. The threshold was selected because there is no evidence to suggest that MAP can survive HTST pasteurization at this initial concentration. The goal of risk mitigation and bulk-milk quality assurance as outlined by Weber et al. (2008) is to focus efforts on reduction of MAP concentration in the bulk tank rather than on complete eradication of the pathogen from the tank or farm environment.

The routes of bulk-milk contamination with MAP may be categorized as either internal or environmental. In the internal route, MAP is shed directly into milk by an infected host, such as through mobilized, MAPpositive macrophages resulting from supramammary lymph node infection or bacteremia (Sweeney et al., 1992). If the source originates outside the mammary gland, the route of contamination is said to be environmental, and MAP may enter the bulk milk by means of fecal contamination or airborne particles (Eisenberg et al., 2013). Additionally, several studies have noted the survival of MAP in a variety of water sources traced to on-farm usage (e.g., Whan et al., 2001; Whittington et al., 2005; Pickup et al., 2006). Contaminated water supplies may represent a source of MAP transmission from cattle to humans, by direct consumption, food incorporation, or the washing of areas contacting food (Whan et al., 2001). It seems plausible that wash water could represent another means of environmental contamination of bulk milk; if the water used for cleaning milking equipment (or udders before milking) contains MAP, the bulk tank may become adulterated.

Traditionally, diagnostics to identify MAP in milk target either MAP antibodies (ELISA) or the organism itself (culture and PCR). An ELISA-positive status likely indicates some type of internal-route shedding into the bulk tank: either infected animals are shedding antibodies alone or are contributing both antibodies and MAP itself. Singh et al. (2007) recorded 84.6\% agreement between individual milk ELISA and milk culture results from the same animals; hence, a positive ELISA is often indicative of internal-route MAP shedding. However, a bulk tank PCR-positive status may represent internal, environmental, or combined routes of contamination.

Of particular interest, then, are farms presenting discordant bulk-milk PCR and ELISA results; on such farms, conclusions may be drawn regarding the routes of contamination. In a recent study, Wilson et al. (2010) noted only moderate agreement between ELISA and PCR in bulk-milk samples, with 113 of 241 samples taken from MAP-positive herds presenting discordant results. Although paired high bulk-tank ELISA and positive PCR results suggest the internal route, possibly with high within-herd prevalence, the effect of environmental contamination cannot be excluded. Bulk tanks that are PCR-negative with high ELISA titers may reveal herds with sufficient hygienic practices in place to restrict environmental contamination despite MAP-positive herd status. Conversely, a low bulk-milk ELISA and positive PCR may imply environmental contamination rather than internal-route shedding of MAP. We hypothesize that herds with this latter combination are those with only a few MAP-positive cows that nevertheless contaminate the bulk tank via the environmental route. Differences in management between these categories of farms may identify specific practices that could be useful in developing control programs to reduce MAP bacterial load in the bulk tank. An enhanced understanding of the routes of bulk-milk contamination and their interrelationship is an essential preliminary step in the reduction of MAP concentration in the bulk tank.

To date, little is known about the relationship between PCR and ELISA results in bulk milk, especially as it relates to management factors and environmental parameters. The objective of this study was to elucidate the relationship between PCR result and ELISA titer using bulk-tank samples and corresponding cross- 
sectional management data collected from 292 farms in 3 US states.

\section{MATERIALS AND METHODS}

\section{Herd Recruitment, Sample Collection, and Questionnaire Data}

Bulk-tank milk samples and questionnaire results originated from a multi-institutional, collaborative study aimed at obtaining cross-sectional data on MAP risk factors. Samples and complete questionnaires were collected from a total of 292 organic and conventional farms from 3 US states: New York (NY), Oregon (OR), and Wisconsin (WI). The recruitment of these herds has been described previously (Cazer et al., 2013; Cicconi-Hogan et al., 2013; Richert et al., 2013). Briefly, 192 organic farms were chosen with the help of extension agents in each county as well as organizations specializing in organic certification. Licensed conventional farms were sourced from state-specific lists provided by state departments of agriculture and 100 of these were selected based upon proximity to the organic farms. Matching was conducted according to herd size (divided into 3 categories: $20-99,100-199$, or $\geq 200$ adult cows) and organic-to-conventional state ratios. Farms were eligible for inclusion if they had a minimum of 20 lactating cows and if they had been shipping milk (certified organic milk in the case of organic farms) for at least 2 yr before the onset of the study.

The questionnaire, described in detail by Richert et al. (2013) and available online (http://milkquality.wisc. edu/organic-dairies/project-c-o-w/), included elements pertaining to general herd management and hygiene practices, as well as to Johne's-specific management factors. The farms were visited between 2009 and 2011, and bulk-tank samples were collected by project personnel (consisting of researchers from Cornell University, Oregon State University, and the University of Wisconsin). As described in Cicconi-Hogan et al. (2013), the bulk tanks were first agitated for a minimum of 5 min before sample collection. Bulk-milk samples were obtained directly from the tank using sterile dippers and sampling containers and immediately placed on ice. Samples from all 3 states were transported or shipped in coolers to Cornell University (Ithaca, NY) for laboratory analysis. At Cornell, samples were frozen at $-20^{\circ} \mathrm{C}$ until testing could be conducted.

Of the 292 farms included in the study, 2 did not approve of Johne's testing of their bulk milk, and the bulk-milk samples from an additional 3 farms had been used up in previous diagnostics. One additional farm had missing data for a covariate under consideration. The total number of analyzed bulk-tank samples was therefore 286: of these, 97 were from NY, 46 from OR, and 143 from WI.

\section{Extraction of DNA from Milk Samples}

The Tetracore VetAlert Real-Time PCR and extraction kits (Tetracore Inc., Rockland, MD) have previously been validated for use in fecal samples, and preliminary results (unpublished material) suggest that this assay may also function well in milk. We conducted optimization experiments using MAP-spiked negative milk, for both the template preparation and quantitative PCR (qPCR) stages to maximize detection potential. The MAP isolates were kindly provided and cultured by the Sweeney Laboratory at The University of Pennsylvania School of Veterinary Medicine (Kennett Square, PA). We evaluated a variety of beadbeating durations, centrifugation speeds, and milk fractionation patterns. The efficacy of several heat treatments and lysis buffers was also considered. Finally, using spiked milk samples and the optimized extraction protocol, we determined the limit of detection for the qPCR. The final optimized protocol, based upon steps outlined by Herthnek et al. (2008) is described here in detail.

Frozen samples were stored overnight at $10^{\circ} \mathrm{C}$. Once thawed, a $15-\mathrm{mL}$ volume from each sample was pipetted into a sterile $50-\mathrm{mL}$ tube and centrifuged at 2,547 $\times g$ for $30 \mathrm{~min}$ at $10^{\circ} \mathrm{C}$. The whey fractions were discarded and the remaining cream and pellets subjected to $1.5 \mathrm{~mL}$ of lysis buffer (prepared using $2 \mathrm{~m} M$ EDTA, $400 \mathrm{n} M \mathrm{NaCl}, 10 \mathrm{~m} M$ Tris at $\mathrm{pH} 8$, and $0.6 \%$ SDS; Sigma-Aldrich, St. Louis, MO), $10 \mathrm{~s}$ of vortexing, and $3 \mu \mathrm{L}$ of $10 \mu \mathrm{g} / \mu \mathrm{L}$ proteinase K (Sigma-Aldrich) to dissolve the cream. The mixture was then transferred to disruption tubes (2-mL microcentrifuge tubes with glass beads; Tetracore Inc.), which were filled to the top of the grooves to allow for a reduction in volume following incubation. Disruption tubes were incubated at $56^{\circ} \mathrm{C}$ for $1 \mathrm{~h}$, followed by beadbeating for $5 \mathrm{~min}$ at three-fourths power (approximately 2,700 rpm) using a Mini-Beadbeater 8 (BioSpec Products, Bartlesville, $\mathrm{OK})$, and then centrifuged at $16,000 \times g$ for $10 \mathrm{~min}$. The supernatant and cream fractions were transferred to 2 -mL microcentrifuge tubes containing $100 \mu \mathrm{L}$ of nucleic acid buffer (Tetracore Inc.), taking care to avoid transferring disruption beads. Samples were vortexed and centrifuged at $1,500 \times g$ for $3 \mathrm{~min}$.

Purification of DNA was conducted beginning with the removal of the supernatant and addition of $560 \mu \mathrm{L}$ of Binding buffer (Tetracore Inc.). The full extraction protocol was followed according to the manufacturer's instruction. If necessary, extracted DNA was stored at $10^{\circ} \mathrm{C}$ for less than $48 \mathrm{~h}$. The protocol was implemented on the 286 milk samples. 


\section{Negative and Positive Extraction Controls}

A MAP-negative milk sample was included in each extraction and qPCR and fully processed using a procedure identical to that used for the unknown samples. Strain K-10 [sequenced by Li et al. (2005) and sequence revised by Wynne et al. (2010)] was used as the positive control and was also extracted according to protocol. The K-10 isolates were kindly provided by the Kapur Laboratory at Pennsylvania State University (University Park, PA). The strain was grown on Herrold's egg yolk slants containing mycobactin J (Fisher Scientific, Pittsburgh, PA) and incubated at $37^{\circ} \mathrm{C}$. Colony growth was assessed weekly; after $12 \mathrm{wk}$, colonies were transferred to tubes containing 7H9 broth (Becton Dickinson, Franklin Lakes, NJ) with Middlebrook Oleic Albumin Dextrose Catalase (Hardy Diagnostics, Santa Maria, CA), cycloheximide, Tween 80 (Sigma-Aldrich), and mycobactin J (Allied Monitor Inc., Fayette, MO) and incubated at $37^{\circ} \mathrm{C}$. Contamination checks were conducted weekly by inoculating a loop-full of suspension to chocolate agar plates (Thermo Scientific, Waltham, MA) and monitoring daily for $3 \mathrm{~d}$ for any bacterial growth before discarding. The optical density (OD) at $600 \mathrm{~nm}$ of the K-10 suspension was measured twice weekly following repeated passage of $1 \mathrm{~mL}$ of the surface layer of broth through a 25-gauge needle. Once the OD reached 0.04 , the presence of MAP was confirmed using acid-fast staining. When used in spiking experiments, $1 \mathrm{~mL}$ of the $\mathrm{K}-10$ suspension was added to 10 $\mathrm{mL}$ of milk. The K-10 DNA was extracted following the procedure described above and diluted 1:100.

\section{Real-Time PCR}

The commercial VetAlert Johne's Real-Time PCR (Tetracore Inc.) was used to quantify MAP in the unknown survey samples. The kit includes a premade master mix containing forward and reverse oligonucleotide primers to amplify the $h s p X$ gene, a FAM-labeled probe to generate a fluorogenic signal, Taq polymerase, and facilitating buffers. The positive control included is a synthetic template in liquid form containing a portion of the target $h s p X$ sequence at 25,000 copies per $2.5 \mu \mathrm{L}$. Tris-EDTA buffer (1×; Affymetrix, USB Corporation, Cleveland, $\mathrm{OH}$ ) was used as the no-template control, consistent with the manufacturer's recommendation.

The samples, in addition to positive and negative PCR and extraction controls, were tested using eleven 96-well plates (Life Technologies, Grand Island, $\mathrm{NY}$ ). The test procedure involved pipetting $22.5 \mu \mathrm{L}$ of master mix into each well in addition to $2.5 \mu \mathrm{L}$ of the appropriate template; positive and negative PCR and extraction controls were added to duplicate wells, and the unknown samples were run in triplicates. The cycling program, executed using a StepOne Real-Time PCR System (Life Technologies), included a 10-min enzyme activation step at $95^{\circ} \mathrm{C}$ followed by a 2 -step PCR, which consisted of 45 cycles $\left(95^{\circ} \mathrm{C} \times 15 \mathrm{~s}, 62^{\circ} \mathrm{C} \times 60 \mathrm{~s}\right)$.

Samples were considered positive at a cycle threshold (Ct) of $\leq 38$. Any samples crossing the threshold after this cutoff value were retested in triplicate; such samples were considered negative unless at least 1 retested replicate was positive at an equal or smaller $\mathrm{Ct}$ and at a higher concentration than the standard curve boundary (i.e., 1 gene copy). Runs were considered valid according to Tetracore if the $\mathrm{Ct}$ values for the positive PCR control at 25,000 copies fell within 20 and 26 and if the no-template control did not cross the threshold during the run (www.Tetracore.com). Because qPCR will be analyzed as dichotomous (positive or negative) rather than by copy number, this variable will hereafter be referred to as "PCR" in the statistical analysis and related discussion. This distinction allows for more general discussions that are less directly dependent on the specific methodology used in this particular experiment.

\section{ELISA}

Samples were tested using the commercial ParaChek ELISA kit (Prionics, Zurich, Switzerland) according to the manufacturer's protocol. The ELISA OD for each bulk-tank sample was "corrected" by subtracting the value of the average negative control for the corresponding plate to adjust for interplate variation. The protocol is described in more detail by Cazer et al. (2013).

Positive and negative classifications for ELISA titers are not validated for bulk-tank samples because the controls and cutoffs represent standards for individual animals. The ELISA results in the bulk tank are therefore interpretable as a continuous measurement and may serve as a proxy for the average MAP infection status in the herd. A high titer may indicate either elevated herd-level antibody production, and thus an overall high prevalence of MAP, or a small number of infected cows that nevertheless contribute high concentrations of antibodies to the bulk tank (Cazer et al., 2013). Thus, ELISA OD in the relative scale may be compared with positive and negative PCR outcomes to identify concordance or discordance of results.

\section{Statistical Analyses}

To fully explore the relationship between bulk-tank ELISA (continuous) and bulk-tank PCR (dichotomous), we generated 2 regression models; the questions 
we sought to answer with each model were related but distinct. First, we hoped to determine whether bulktank PCR result could predict ELISA titer after accounting for other variables already known to be related to ELISA, such as season of sampling and protocols for managing MAP-positive cows (Cazer et al., 2013). To address this question, we developed a linear regression model using bulk-tank ELISA OD as the outcome. Second, we attempted to evaluate how management factors affected bulk-milk MAP contamination via the environmental route and whether bulk-tank ELISA titer significantly predicted PCR once environmental parameters were accounted for. For this objective, we treated PCR as the outcome in a binary logistic regression model. All statistical testing was executed using SAS (version 9.4, SAS Institute Inc., Cary, NC). Figures were generated using JMP Pro (version 10, SAS Institute Inc.).

Linear Regression Model. Corrected ELISA OD was modeled as the outcome in a multivariable linear regression using a backward, stepwise selection method (PROC GLMSELECT). In addition to PCR result (positive or negative), seasonality and management procedures for Johne's positive cows were included as potential predictors, based upon a previous published model developed by Cazer et al. (2013). Consistent with this model, the seasonality variable was created using the function $\operatorname{Cos}\left(2 \pi \frac{D a y}{365}\right)$, with "Day" representing the day of the year as a continuous variable ranging from 1 to 365. The "protocols for managing MAP-positive cows" variable was categorized as either "cull immediately," "cull after dry off or calving," "keep," or "never had a positive Johne's test or clinical Johne's disease."

The variables "state" (New York, Oregon, or Wisconsin), "herd size" ( $>200,100-200$, or $<100)$, and "production type" (conventional or organic) were forced to remain in the final model due to the importance of these parameters in determining the study population. The herd size variable was treated as purely categorical, rather than ordinal, to allow for the possibility of a nonlinear relationship between the size of a herd and its bulk-tank MAP status. Parameters were generated using reference coding, and the standard assumption that $\varepsilon_{i j}$ are $N\left(0, \sigma^{2}\right)$ was assessed. The model was of the form

$$
\begin{aligned}
\hat{Y}_{\text {ELISA }}= & \alpha+\hat{\beta}_{1} X_{\text {herdsize }}+\hat{\beta}_{2} X_{\text {herdsize }}+\hat{\beta}_{3} X_{N Y} \\
& +\hat{\beta}_{4} X_{O R}+\hat{\beta}_{5} X_{\text {Conventional }}+\hat{\beta}_{6} X_{P C R}+\hat{\beta}_{k} X_{k}
\end{aligned}
$$

where $\alpha$ is the intercept, $\hat{\beta}_{1}$ through $\hat{\beta}_{k}$ are the leastsquares parameter estimates for the included covari- ates, and $\hat{Y}$ is the predicted value of ELISA based upon the values for the resulting model coefficients; $\hat{\beta}_{1}$ and $\hat{\beta}_{2}$ are the corresponding parameter estimates for herd size (of $>200$ and 100-200, respectively, with a herd size of $<100$ serving as the reference level); $\hat{\beta}_{3}$ and $\hat{\beta}_{4}$ correspond to state (NY and OR, respectively, with WI as the reference level), and $\hat{\beta}_{5}$ corresponds to production type (specifically to conventional farms, with organic farms as the reference level); $\hat{\beta}_{6}$ is the parameter estimate for positive PCR result (with a negative result serving as the reference). The $X_{k}$ term represents additional significant predictor variables and their associated coefficients $\left(\hat{\beta}_{K}\right)$. For the linear model, these potential predictors were season of sampling and protocols for managing MAP-positive cows.

Logistic Regression Model. Variables from the management questionnaire were chosen based upon a potential association with MAP prevalence. Univariable analyses were then conducted as a method of variable selection for the logistic model: the relationship of bulk-tank PCR to continuous variables was evaluated using $t$-tests (PROC TTEST), whereas the relationship of bulk-tank PCR to binary or polychotomous categorical variables was evaluated using Chi square or Fisher's exact tests (PROC FREQ). Variables associated with $\mathrm{PCR}$ and demonstrating statistical significance at the $P<0.05$ level were marked for inclusion in the multivariable model. No adjustments were made for multiple testing to provide a sufficiently liberal threshold for inclusion. As in the linear model, state (NY, OR, or WI), herd size $(>200,100-200$, or $<100)$, and production type (conventional or organic) were forced to remain in the model due to the role of these variables in farm selection. Bulk-tank PCR was considered as a binary variable, and the log odds of "PCR positive" were modeled using logistic regression (PROC LOGISTIC) with a penalized maximum likelihood function (Firth's bias adjustment) to correct for quasi-separation of data. Two-way interactions were tested and included in the model providing they offered significant improvement to the model fit (as evaluated using likelihood ratio tests.) The overall fit of the model was evaluated using the Hosmer-Lemeshow goodness-of-fit test. A biologically relevant increase in ELISA OD of 0.1 units was used in odds ratio calculations by exponentiating 0.1 times reference-coded parameter estimates. The form for the logistic model was as follows:

$$
\begin{aligned}
\ln \left(\frac{P_{P C R \text { positive }}}{P_{P C R \text { negative }}}\right)= & \alpha+\hat{\beta}_{1} X_{\text {herdsize }}+\hat{\beta}_{2} X_{\text {herdsize }} \\
100-200 & \\
& +\hat{\beta}_{3} X_{N Y}+\hat{\beta}_{4} X_{O R}+\hat{\beta}_{5} X_{\text {Conventional }} \\
& +\hat{\beta}_{6} X_{E L I S A}+\hat{\beta}_{k} X_{k},
\end{aligned}
$$


where $\ln \left(\frac{P_{P C R} \text { positive }}{P_{P C R} \text { negative }}\right)$ represents the natural log of the odds of PCR positivity in the bulk tank (the probability of being PCR positive divided by the probability of being PCR negative) based upon the values for the logistic model parameters; $\alpha$ is the intercept, and $\hat{\beta}_{1}$ through $\hat{\beta}_{k}$ are the Firth-adjusted parameter estimates for the included covariates, estimated by maximum likelihood. $\hat{\beta}_{1}$ and $\hat{\beta}_{2}$ are the corresponding parameter estimates for herd size (of $>200$ and 100-200, respectively, with a herd size of $<100$ serving as the reference level); $\hat{\beta}_{3}$ and $\hat{\beta}_{4}$ correspond to state (NY and OR, respectively, with WI as the reference level), and $\hat{\beta}_{5}$ corresponds to production type (specifically to conventional farms, with organic farms as the reference level); $\hat{\beta}_{6}$ is the parameter estimate for corrected ELISA OD in the bulk tank. The $X_{k}$ term represents any additional significant predictor variables or interactions between included predictor variables, and their associated coefficients $\left(\hat{\beta}_{K}\right)$. In the logistic model, these potential predictors were nutritionist use, fall housing of preweaned heifers, spring housing of preweaned heifers, presence of scours in calves, and feeding calf starter.

\section{RESULTS}

\section{Optimization of Template Preparation and Real-Time PCR}

We conducted a variety of optimization experiments using MAP-spiked negative milk. First, 3 beadbeating durations were compared using a Mini-Beadbeater and followed up by DNA purification and qPCR. The durations tested were 1 min (as in Herthnek et al., 2008), 5 min (as in Odumeru et al., 2001), and an intermediate duration of $2 \mathrm{~min}$. A range of $\mathrm{OD}$ values $(0.04,0.13$, $0.24,0.34$, and 0.43 ) was implemented for each duration to account for the potential influence of quantity on the latency to disrupt the cells. The differences between the durations were found to be significant in a mixed-design ANOVA $(P=0.0002)$. For all OD values tested, the $\mathrm{Ct}$ values for 1 min were universally higher (corresponding to lower quantities of MAP) than those for 2 and 5 min in the corresponding samples $(P=0.003$ and $P=$ 0.0002 , respectively, in Tukey's honestly significantly different post hoc tests). There was no statistical difference between the 2- and 5-min durations. Presented with the choice between 2 and 5 min, we selected 5 min of beadbeating; this decision was based upon a numerically lower $\mathrm{Ct}$ average and the use of $5 \mathrm{~min}$ in several studies in which the cream and pellet were combined (Odumeru et al., 2001; Gao et al., 2005).
Consistent with the findings of other studies (notably Gao et al., 2005, 2007; and Herthnek et al., 2008), we found detectable levels of MAP in both the cream and pellet layers and the best overall signal when the pellet and cream were pooled during template preparation. Both hexadecylpyridinium chloride (Sigma-Aldrich) and a lysis buffer (containing EDTA, $\mathrm{NaCl}$, Tris, and SDS) in combination with proteinase K (Sigma-Aldrich) were evaluated for their ability to maximize recovery of MAP from the cream, with the latter combination providing the best signal. Several heat treatments were also assessed, although none provided a consistent improvement.

In optimization studies using MAP-spiked negative milk, the qPCR performed well and was able to detect dilutions of $10^{-3.5}$ (corresponding to $82.3 \mathrm{cfu} / \mathrm{mL}$ ) in all plates tested, with an average reported copy number of $3.655 \pm 0.028$. A dilution of $10^{-4.5}(8.23 \mathrm{cfu} / \mathrm{mL})$ amplified in approximately half of the wells tested, although this concentration fell outside of the boundaries established by the standard curve (i.e., the copy number was $<1$ ).

\section{Questionnaire}

With regard to self-reported Johne's-related responses and perception of Johne's disease, 56.5\% of farms in the study had observed at least one "clinical Johne's case" since the year 2000; however, only $2.4 \%$ of these farms reported confirmation of these cases by veterinary diagnosis. Clinical cases were most often identified by farm staff if the animal showed signs of loose manure $(89.7 \%)$ or poor body condition $(69.1 \%)$. At the time of sample collection, $27.7 \%$ of farms were participating in a Johne's control program and 16.1\% had a written plan for Johne's disease management. Conventional farms were more likely to participate in a Johne's control program compared with organic farms $\left(\chi_{(\mathrm{df}=1)}^{2}=9.366, P=0.002\right)$ and were more likely to have a written plan for Johne's disease management $\left(\chi_{(\mathrm{df}=1)}^{2}=3.925, P=0.048\right)$

\section{ELISA}

The ELISA tests were conducted on 288 samples. Corrected optical densities $\left(\mathrm{OD}_{600}\right)$ ranged from -0.098 to 0.368 after subtraction of the negative control, with an average OD of $-0.023 \pm 0.003$. The distribution of ELISA OD results is shown in Figure 1.

\section{Real-Time PCR}

Real-time PCR was conducted on 286 samples. All runs were valid according to the criteria specified by 


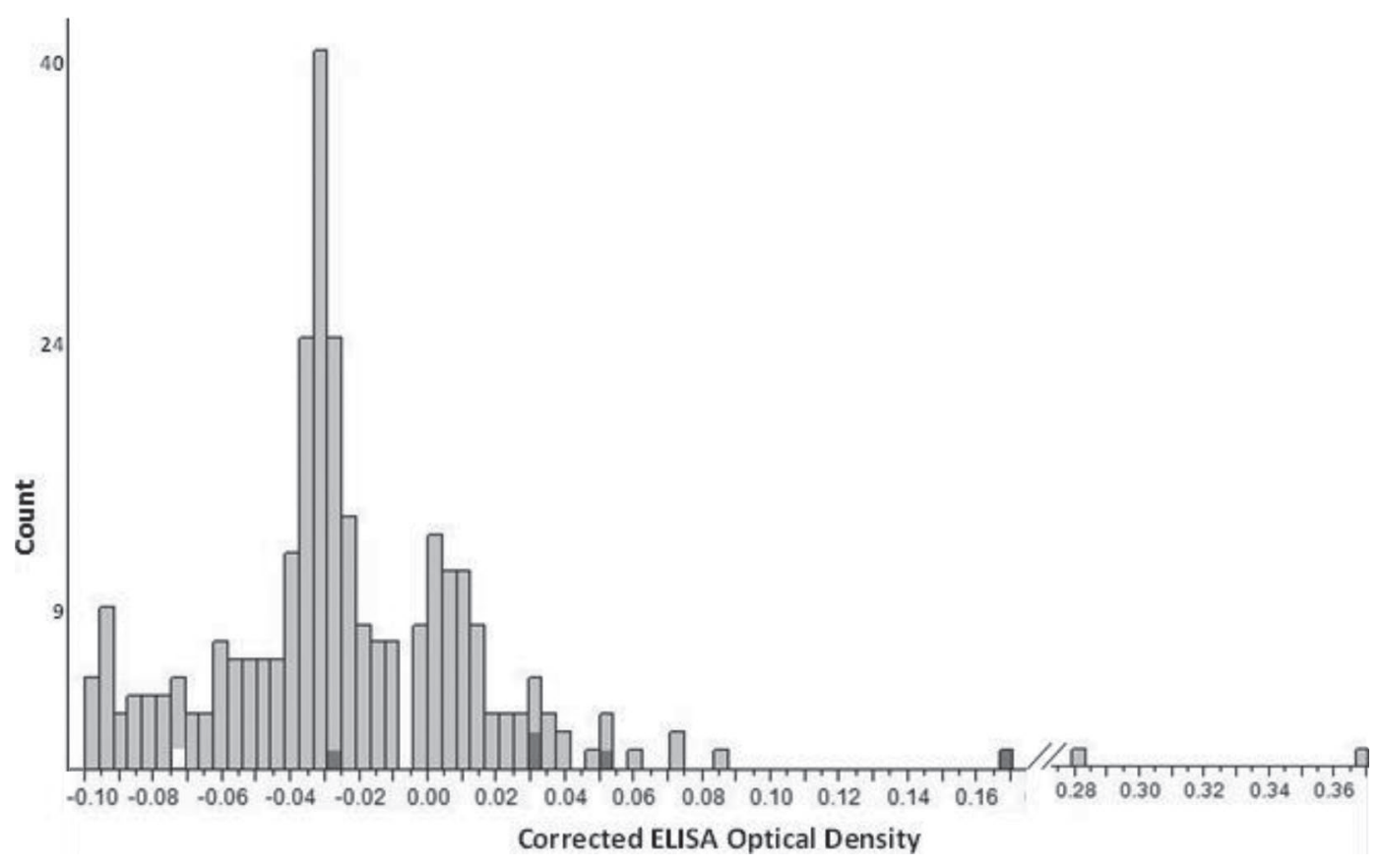

Figure 1. Frequency histogram of corrected ELISA optical densities (OD) in bulk-tank milk, with count on the y-axis and ELISA OD on the x-axis. Farms with PCR-positive bulk milk are marked in dark gray (organic farms) or white (conventional farm); PCR-negative farms are represented by light gray.

Tetracore (www.Tetracore.com), and the negative extraction control did not amplify during any of the runs. The mean $\mathrm{R}^{2}$ and efficiency values were $0.992 \pm 0.002$ and $96.441 \pm 0.812 \%$, respectively. There were 6 positive samples corresponding to a mean copy number of $3.660 \pm 1.522$.

The farms with PCR-positive bulk tanks may be categorized in terms of their production type, herd size, and state. Five were organic: 3 from NY with herd sizes $<100,1$ from NY with herd size $>200$, and 1 from WI with a herd size of $<100$. There was an additional PCR-positive bulk tank from an NY herd, which was a nongrazing conventional farm with a herd size of $>200$. The relationship between bulk-tank PCR positivity and bulk-milk ELISA titer is illustrated in Figure 1, which is coded according to production type.

\section{Linear Regression Model}

The final linear regression model with corrected ELISA OD as the outcome variable was significant overall $(F=13.07, P<0.0001)$, indicating a rejection of the global null hypothesis that no $\beta$ estimate is different from 0 . The residuals were approximately normally distributed and centered around 0, although there were several outliers. In any case, the GLMSELECT procedure may provide valid approximations in spite of minor normality violations (SAS User's Guide; SAS Institute Inc.). None of the variables forced into the model (herd size, production type, or state) was a significant predictor of ELISA. The cosine seasonality curve remained highly significant $(P<0.0001)$, retaining the peak in OD in the summer months consistent with the previous model developed by Cazer et al. (2013). The variable "protocols for managing MAP-infected cows" was no longer significantly associated with ELISA OD and was removed from the model. Notably, PCR was significant $(P=0.002)$ and therefore conserved in the final model, which may be expressed as

$$
\begin{aligned}
\hat{Y}_{\text {ELISA }}= & \alpha+\hat{\beta}_{1} X_{\text {herdsize }}+\hat{\beta}_{2} X_{\text {herdsize }}+200 \\
& +\hat{\beta}_{3} X_{N Y} \\
& +\hat{\beta}_{4} X_{O R}+\hat{\beta}_{5} X_{\text {Conventional }}+\hat{\beta}_{6} X_{\text {cos_season }}+\hat{\beta}_{7} X_{\mathrm{PCR}} .
\end{aligned}
$$

The values for the intercept and for $\hat{\beta}_{1}$ through $\hat{\beta}_{7}$ and their associated significance levels are provided in Table 1.

\section{Univariable Analyses}

The variables found to be significantly associated with PCR result following univariable analyses were ELISA (corrected OD), presence of scours in calves, housing for preweaned heifers (in the fall and spring), feeding calf starter, and nutritionist use. These variables were considered appropriate potential predictors for the logistic regression model because of their pre- 
Table 1. Linear regression model with corrected bulk-milk ELISA optical density as the outcome; referencecoded parameter estimates $(\hat{\beta})$, associated SE, $t$ ratios, and significance levels are shown

\begin{tabular}{|c|c|c|c|c|}
\hline Term & $\hat{\beta}$ & $\mathrm{SE}$ & $t$ ratio & $P>|t|$ \\
\hline Intercept & -0.028 & 0.004 & -6.85 & $<0.001$ \\
\hline \multicolumn{5}{|l|}{ State } \\
\hline New York & -0.001 & 0.006 & -0.15 & 0.884 \\
\hline Oregon & -0.001 & 0.008 & -0.15 & 0.883 \\
\hline Wisconsin & Reference & & & \\
\hline \multicolumn{5}{|l|}{ Herd size } \\
\hline$>200$ & -0.012 & 0.008 & -1.43 & 0.154 \\
\hline $100-200$ & -0.007 & 0.007 & 0.88 & 0.381 \\
\hline$<100$ & Reference & & & \\
\hline \multicolumn{5}{|l|}{ Production type } \\
\hline Conventional & 0.005 & 0.005 & 0.89 & 0.374 \\
\hline Organic & Reference & & & \\
\hline \multicolumn{5}{|l|}{ PCR result } \\
\hline Positive & 0.054 & 0.017 & 3.13 & $0.002^{*}$ \\
\hline Negative & Reference & & & \\
\hline \multicolumn{5}{|l|}{ Seasonality } \\
\hline Cosine $\left(2 \pi \frac{d a y}{365}\right)$ & -0.029 & 0.003 & -8.62 & $<0.0001^{*}$ \\
\hline
\end{tabular}

* Significant at $P<0.05$.

viously documented relationship to MAP prevalence. Significance levels from the preliminary univariable analyses are shown in Table 2 .

\section{Logistic Regression Model}

The variables selected in the univariable analyses were considered eligible predictors for inclusion in the Firth-corrected, binary logistic regression model with the logit of PCR positive as the outcome. The variables herd size $(<100,100-200$, or $>200)$, production type (organic or conventional), and state (NY, OR, or WI) were forced to remain in the model.

The corrected parameter estimates are shown in Table 3 in addition to associated significance levels, odds ratio estimates, and $95 \%$ confidence intervals. The overall model was significant according to the likelihood ratio test, $\left(\chi_{(\text {df } 7)}^{2}=24.477, P<0.001\right)$, suggesting that an improved fit is provided relative to a model containing the intercept alone. The model fit the data well as indicated by the Hosmer and Lemeshow goodness-of-fit test $\left(\chi_{(\text {df } 8)}^{2}=2.579, P=0.958\right)$, and the quasi-nonconvergence was successfully controlled by means of the bias correction.

Of the forced-entry predictors, there was a significant effect of state; specifically, the model demonstrated an increase in the odds of being PCR positive in the bulk tank for farms in NY compared with WI. There was also an effect of herd size, with the largest herd size category $(>200)$ showing an increased odds relative to the smallest $(<100)$. Of the other candidate predictors, ELISA OD was retained and an interaction was uncovered between production type and ELISA OD. The interaction provided a significant improvement to the model fit, as evaluated using a likelihood ratio test for nested models.

Figure 2, representing the interaction between bulkmilk ELISA and production type, reveals that, for or-

Table 2. Candidate predictors for the logistic regression model $^{1}$

\begin{tabular}{|c|c|c|c|c|}
\hline Variable & Description & $\begin{array}{l}\text { Variable } \\
\text { type }\end{array}$ & Test & $P$-value \\
\hline Nutritionist & Use of nutritionist (yes/no) & Binary & Fisher's exact & 0.028 \\
\hline Spring housing & $\begin{array}{l}\text { Preweaned heifers housed in a group area (multiple animal area, } \\
\text { pasture/drylot) compared with an individual or restricted area } \\
\text { (hutches, individual animal area, or tied in a barn) }\end{array}$ & Binary & Fisher's exact & 0.012 \\
\hline ELISA & Corrected ELISA optical density & Continuous & $t$-test & $\begin{array}{c}P<|t| \\
0.004\end{array}$ \\
\hline
\end{tabular}

${ }^{1}$ The variables shown were significantly related to bulk-milk PCR result $(P<0.05)$ via the specified univariable analyses and considered eligible for inclusion in the logistic regression model. 
ganic farms, as ELISA titer increases, so too does the value of the linear predictor, which is directly related to the probability of bulk-milk PCR positivity $\left[P(P C R+)=\frac{1}{1+e^{-(\text {linear predictor })}}\right]$. The figure also shows a weaker relationship between the linear predictor and bulk-tank ELISA titer on conventional farms.

The final model, as detailed in Table 3, was of the form

$$
\begin{aligned}
\ln \left(\frac{P_{P C R \text { positive }}}{P_{P C R \text { negative }}}\right)= & \alpha+\hat{\beta}_{1} X_{\text {herdsize }}+\hat{\beta}_{2} X_{\text {herdsize }} \\
& +\hat{\beta}_{3} X_{N Y}+\hat{\beta}_{4} X_{\text {OR }}+\hat{\beta}_{5} X_{\text {Conventional }} \\
& +\hat{\beta}_{6} X_{\text {ELISA }}+\hat{\beta}_{7} X_{\text {ELISA Conventional }},
\end{aligned}
$$

where $\hat{\beta}_{7}$ represents the coefficient for an interaction between bulk-milk ELISA and production type (with organic farms again serving as the reference level for the interaction).

\section{DISCUSSION}

Our data demonstrate a strong relationship between ELISA and PCR results in bulk-tank milk. In the logistic model, ELISA was an important predictor of PCR status, particularly on organic farms, even after consideration of more than 300 relevant environmental and management factors. Additionally, and as expected, PCR significantly predicted ELISA in the linear model after controlling for seasonality.

Specifically, these results point to a substantial level of concordance between positive PCR outcomes and high ELISA OD in the same bulk-tank samples. Although the possibility of environmental contamination cannot be excluded on these farms, paired high bulk-tank ELISA and positive PCR results allude to the internal route, likely with a high prevalence. Direct shedding by late-stage MAP-infected cows may therefore be the dominant source of bulk-milk contamination on these farms, because antibody production occurs mainly in progressors (Schukken et al., 2015) and in the later stages of infection when MAP is systemically present (Streeter et al., 1995). It may be assumed that such late-stage MAP-infected animals are shedding MAP directly into their milk, because there is a strong trend for an increased ELISA titer with an increased shedding category (Schukken et al., 2015) and a reported high agreement between milk ELISA results and milk culture from individual animals (Singh et al., 2007).

Although studies such as Wilson et al. (2010) have called attention to a certain level of discordance between PCR and ELISA diagnostics in bulk-tank milk samples, this relationship hinges on a positive/negative designation for ELISA results. Because the standards on ELISA plates are based upon individual-animal lev-

\begin{tabular}{|c|c|c|c|c|c|c|}
\hline Term & $\begin{array}{l}\text { Parameter } \\
\text { estimate }\end{array}$ & $\mathrm{SE}$ & Wald $\chi^{2}$ & $P>\chi^{2}$ & $\widehat{\mathrm{OR}}$ & $\begin{array}{l}95 \% \text { Wald CI } \\
\text { for } \widehat{O R}\end{array}$ \\
\hline Intercept & -5.293 & 1.153 & 21.063 & $<0.001$ & - & - \\
\hline \multicolumn{7}{|l|}{ State } \\
\hline Oregon & -0.277 & 1.726 & 0.026 & 0.873 & 0.758 & $0.026,22.341$ \\
\hline Wisconsin & Reference & - & - & - & - & - \\
\hline \multicolumn{7}{|l|}{ Herd size } \\
\hline$<100$ & Reference & - & - & - & - & - \\
\hline \multicolumn{7}{|l|}{ Production type } \\
\hline Conventional & -0.454 & 0.905 & 0.252 & 0.616 & - & $-^{2}$ \\
\hline Organic & Reference & - & - & - & - & - \\
\hline \multicolumn{7}{|l|}{ ELISA } \\
\hline (corrected optical density) & 36.312 & 10.356 & 12.295 & $0.001^{*}$ & - & $-^{2}$ \\
\hline \multicolumn{7}{|l|}{ ELISA $\times$ production type } \\
\hline
\end{tabular}
els, antibodies in the bulk tank may be diluted beyond

Table 3. Firth-corrected parameter estimates for the logistic regression model with bulk-tank PCR result as the outcome ${ }^{1}$

${ }^{1}$ The log odds of being PCR positive in the bulk tank are modeled. Also shown are the standard errors of the estimate, Wald Chi-squared statistics with significance levels, and odds ratio estimates $\widehat{(\mathrm{OR})}$ with $95 \%$ Wald CI. Odds ratios, computed based upon specified reference levels, are significant if the corresponding Wald CI does not include 1.00.

${ }^{2}$ The presence of a significant interaction precludes computation of main-effect odds ratios.

${ }^{3}$ The odds ratios for the interaction were calculated based upon a biologically relevant 0.1-unit increase in ELISA optical density.

* Significant at $P<0.05$. 


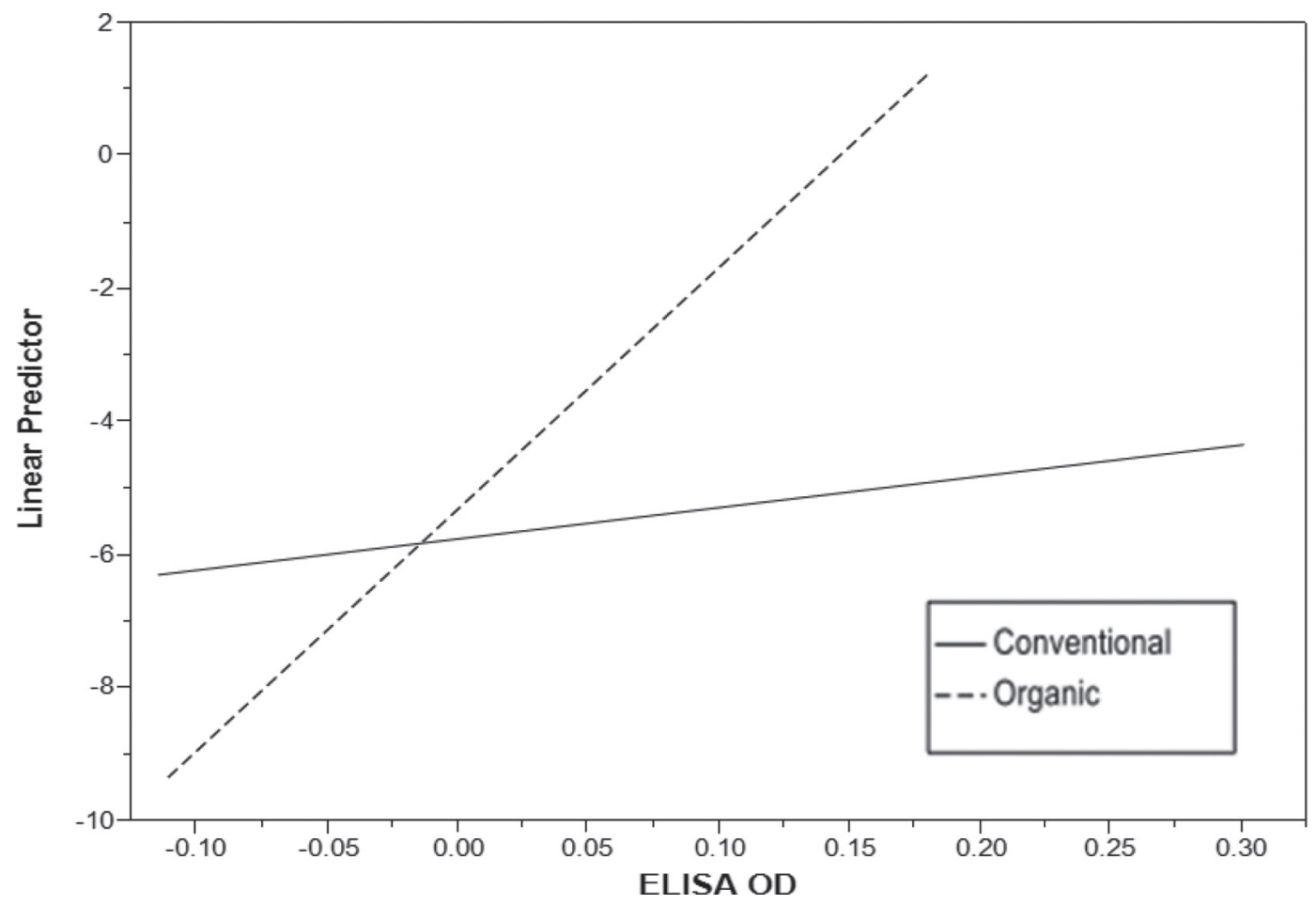

Figure 2. Representation of the interaction between bulk-milk ELISA optical densities (OD) and production type (organic vs. conventional) on bulk-milk PCR status. The figure shows the linear predictor on the $\mathrm{y}$-axis and ELISA OD on the x-axis. Values of the linear predictor were computed for the reference levels Wisconsin and herd size $<100$ at the observed ELISA titers.

the "positive" threshold yet still indicate a low prevalence of MAP antibody-positive cows.

Nevertheless, the concordance in our data does not preclude discordance on some farms; despite the demonstrable agreement between PCR positives and high ELISA titers in our bulk-tank samples, the majority of high ELISA farms were PCR negative. Although falsely high bulk-milk ELISA cannot be completely ruled out, the high ELISA-negative PCR profile suggests a general presence of MAP in these herds but little direct excretion into the bulk tank. Importantly, high ELISA farms that are PCR negative indicate an apparent lack of environmental contamination; the implication is that farms in NY, OR, and WI are largely able to maintain proper hygiene and management practices to ensure a low bacterial load in the bulk tank and thus low-risk milk, despite an estimated high prevalence.

The slight positive skew of the corrected bulk-milk ELISA titers (as apparent in Figure 1) suggests that the majority of farms have ELISA values at the lower end of the spectrum. The low ELISA farms are almost universally PCR negative in the bulk tank, a combination that is ideal with respect to bulk-milk status. An anomaly in the data set was a bulk-tank PCR-positive farm with a relatively low ELISA titer of -0.072 (roughly the 10th percentile). Farms with this PCR positive-low ELISA profile may house a small number of infected cows that nevertheless contaminate the bulk tank by means of the environmental route (Cazer et al., 2013), because internal-route contamination with MAP would likely be accompanied by antibody secretion. It is conceivable that early-stage, intermittently shedding cows may be shedding the bacteria directly into milk before the development of detectable antibody levels. However, this effect would be more plausibly observed in individual animals rather than at the herd level. In order for such a herd-level effect to be apparent, the majority of the lactating herd would need to be in the same phase of infection and exhibit similar shedding patterns into milk. This likelihood seems low, as van Schaik et al. (2003) noted that kinetics ELISA results (for serum) were highly variable at the cow level. The between-cow variability is likely present with respect to milk antibodies as well. Although a positive fecal culture is generally understood to precede a positive ELISA (van Schaik et al., 2003), the latency to develop an antibody response following the onset of shedding is usually restricted to several months. Little information exists on the sequential relationship of direct shedding of MAP into milk and the humoral immune response, 
but it is improbable that the animals in a herd would be in precisely the same phase relative to milk antibody production and direct shedding into milk.

Bulk-milk ELISA results appear to be beneficial for identifying high-risk farms for potential zoonotic transmission (particularly in the event that further evidence links MAP to Crohn's development, as the zoonotic potential of MAP has not been proven). However, ELISA is not a perfect predictor of bulk-milk contamination, as demonstrated in previous work (Van Weering et al., 2007) and as evidenced by the farms in the current study displaying high levels of MAP antibodies in the bulk tank without the apparent presence of the pathogens themselves. A combination of bulk-tank ELISA and PCR results should therefore provide a better tool for potential zoonotic risk assessment than ELISA alone. The Tetracore VetAlert Real-Time PCR and extraction kits (www.Tetracore.com) appear to be useful diagnostics that function well not only for fecal samples (Alinovi et al., 2009) but also for bulk-tank milk samples. Successful optimization of the template preparation methods and beadbeating durations relied on the observation that MAP fractionates to both the pellet and cream layers (Gao et al., 2007; Herthnek et al., 2008). Following optimization, we were able to use the Tetracore kits to reliably detect MAP concentrations $>10^{-4.5}$ (corresponding to $8.23 \mathrm{cfu} / \mathrm{mL}$ ) in bulktank samples, with the caveat that optimization was conducted using spiked milk rather than with positive farm samples.

Variation exists regarding the rate of bulk-tank positivity as detected by PCR. At one end of the spectrum, Khol et al. (2013) found a 0\% positive rate in bulktank samples throughout their study of low-prevalence Austrian herds. The rate of $2.10 \%$ sample positivity for our PCR results (with a $5.16 \%$ rate for NY farms) appears consistent with rates reported in numerous other studies, including Bosshard et al. $(2006 ; 3.00 \%)$ and Cetinkaya et al. (1996; 3.50\%), although several studies have reported a higher prevalence based on bulk-tank PCR. For example, Corti and Stephan (2002) reported a $19.7 \%$ positive rate using IS900 PCR for bulk-tank samples collected from different regions in Switzerland, with a region-dependent prevalence ranging from 1.7 to $49.2 \%$.

The bulk-tank PCR-positive rate is undoubtedly affected by region, method, and gene target used for detection. There are specificity issues linked to PCR based on the IS900 target because highly homologous sequences have been identified in other environmental mycobacteria (Bosshard et al., 2006; Slana et al., 2008). The Tetracore qPCR targets the $h s p X$ gene, which is thought to be involved in intracellular survival and occurs as a single copy in the MAP genome; its presence in single-copy form facilities quantitation. Compared with $I S 900$, the $h s p X$ target is less prone to false-positive results, improving reliability (Slana et al., 2008). When used on fecal samples, the Tetracore test has a specificity of $97 \%$ (Alinovi et al., 2009). Because the test is commercially available, it fits the criteria of being a simple and rapid option for herd-level testing.

Milk-filter testing has also been explored as a potential herd-level indicator of MAP presence. Slana et al. (2012) concluded that qPCR testing of milk filters could be beneficial for identifying MAP, in addition to providing a rough prevalence estimate for a given farm. This technique could be classified as both inexpensive and simple, because commercial test kits may be used and the filter is readily accessible. Yet, the presence of MAP on the milk filter likely does not correlate perfectly with its presence in bulk milk. Given the practice of feeding waste milk to calves and the potential relationship of MAP and human Crohn's disease, evaluation of the bulk milk itself may prove more informative.

Used in combination with bulk-milk ELISA, bulkmilk PCR results may provide a useful indication of the MAP status of the herd and the routes of contamination on a given farm. Although ELISA OD has been considered an imprecise tool to evaluate the status of individual animals, the titers do indeed appear to be useful indicators of overall herd MAP shedding level. Lombard et al. (2006) found that the herd-level sensitivity for the Parachek ELISA was comparable to that of serum ELISA and ranged from 56 to $83 \%$ (when fecal culture was used as a reference for infection status). The application of ELISA for bulk-tank milk has not yet been extensively explored, but some information can be obtained on the sensitivity and specificity of bulk-milk diagnostics. Van Weering et al. (2007) were able to test these parameters for the Pourquier ELISA using bulk-tank samples from herds with known seroprevalence. In herds with $3 \%$ or greater seroprevalence, the sensitivity was $85 \%$ and the specificity $96 \%$ (using a cutoff revised for bulk-tank samples.) Thus, the positive and negative predictive values were, respectively, 67 and $94 \%$. Nielsen et al. (2000) reported $97.1 \%$ sensitivity and $83.3 \%$ specificity for a milk ELISA (using antibodies from Allied Monitors, Fayette, MO) adapted for bulk-tank use. Despite the high sensitivity, Nielsen et al. (2000) argued that the diagnostic test had limitations due to the ability of small variations in the cutoff to dramatically affect the estimated prevalence. This observation provides support for the interpretation of bulk-tank ELISA as a continuous variable to scale the risk of MAP infection in the herd, rather than in relation to a cutoff level.

There is some reported variation in the sensitivity and specificity of milk ELISA based upon the method 
used, and sensitivity may be increased through the use of modified protocols (Cazer et al., 2013). McKenna et al. (2005) compared absorbed and unabsorbed indirect assays evaluated against tissue and fecal cultures. The unabsorbed ELISA had increased sensitivity but this advantage came at the expense of lower test accuracy and specificity. The negotiation and compromise between sensitivity and specificity can be obviated in part by interpreting the ELISA values as numerical rather than dichotomous, indicative of the probability of herd MAP infection. However, it is difficult to completely avoid the sources of variability resulting from the kit or method used for milk ELISA, particularly with respect to a disease such as Johne's, because no "gold standard" exists for reference.

Bulk-tank PCR results on high ELISA farms may depend upon whether the farm is organic or conventional: we uncovered a potential interaction in the logistic model between ELISA and production type. The interaction revealed a strong linear relationship between ELISA titer and the logit of PCR positivity for organic farms and essentially an absence of a relationship between bulk-milk ELISA titer and PCR result for conventional farms. According to the logistic model, a biologically relevant increase of 0.1 units in ELISA OD on organic farms resulted in a 37.76 multiplicative increase in the odds of being PCR positive in the bulk tank. For conventional farms, the same 0.1-unit change would yield instead an increase of only 1.60. This may suggest that, in our data, conventional farms with a high MAP prevalence are better equipped to temper environmental contamination. Indeed, conventional farms in our study were more likely to participate in a Johne's control program and have a written plan for Johne's disease management, compared with organic farms.

Another possible explanation may involve the age and retention rate of animals. Stiglbauer et al. (2013) concluded that the mean percentage of first-lactation animals was lower on organic farms compared with conventional herds. This study appears to affirm the notion that organic farms tend to retain older animals and perhaps have an increased risk of age-related disease (Stiglbauer et al., 2013) As Johne's disease is progressive, subclinically infected cows retained for a longer period may become heavy shedders or develop clinical Johne's and thus increase the odds of both direct shedding of MAP into milk and environmental contamination. The interaction is graphically represented in Figure 2. For organic farms, bulk-tank ELISA titer increased alongside values of the linear predictor, which were related to the probability of PCR positivity in the bulk tank. On conventional farms, on the other hand, the values for the linear predictor, and thus the associ- ated probabilities, remained relatively constant across the differing levels of ELISA titer. Consequently, a high ELISA titer seems to better predict positive bulk-tank PCR status on organic compared with conventional farms.

The interaction was included in the model due to its significance level; however, as shown in Figure 1, all but one of the PCR-positive bulk tanks were from organic farms. The absence of a relationship between bulk-milk ELISA and PCR results for conventional farms may reflect a lack of data on this subgroup rather than a true difference. There was nevertheless a strong relationship between ELISA titer and PCR status in bulk milk, at least with respect to organic farms, and the possibility of a true interaction cannot be excluded. Moreover, this is not the case of opposing PCR-ELISA relationships observed for the different farm types: the relationship between bulk-tank ELISA and PCR for both conventional and organic farms manifests in the same direction (i.e., both slopes are positive). Therefore, the main conclusions regarding the PCR-ELISA relationship in the bulk tank remain the same regardless of whether the interaction is included in the model.

As indicated, all but one PCR-positive bulk tanks were from organic farms. Although the main effect of production type was nonsignificant in our logistic model, it should not necessarily be interpreted as such, due to the presence of an interaction. Conventional farms may be able to keep prevalence low by the use of milk replacer rather than pasteurized or unpasteurized milk from the herd. There is no approved commercially available organic milk replacer (Stiglbauer et al., 2013). Stiglbauer et al. (2013) noted that management on conventional and organic dairies differed insofar as conventional farms made use of outside resources such as the DHIA and veterinarians (also observed by Zwald et al., 2004) much more frequently than did organic farms. Additionally, conventional farms more often called in nutritionists, and cattle on these farms were fed almost twice as much grain as were cattle on organic dairies (Stiglbauer et al., 2013). In our study, the univariable analyses seemed to suggest a relationship between nutrition (in particular, the use of a nutritionist and the provisioning of calf starter) and negative bulk-tank PCR status. It has been suggested that calves are more likely to sustain MAP infection if they have not been provided with adequate nutrition (Doyle, 1956). Thus, Sorge et al. (2012) hypothesized that the combination of poor nutrition, which would heighten calf susceptibility, and exposure to fecal pathogens may lead to an increase in herd MAP prevalence. The potential connections between MAP infection, nutrition, and production type are interesting to consider and may certainly be explored further. 
With respect to geographical distribution, all but one of the farms in our data set with PCR-positive bulk milk were located in New York State. Moreover, the NY location was significantly related to bulk-tank PCR status according to the logistic model. When compared with WI herds, farms in NY had 11.59 times greater odds of being PCR positive in the bulk tank. This finding does not align with results of previous studies indicating that a positive MAP status (Wells and Wagner, 2000) or environmental contamination with MAP (Lombard et al., 2006) is more common in Midwestern herds. However, the diagnostics used by Wells and Wagner (2000) were serological ELISA on individual animals, paired with prior knowledge regarding clinical signs in the herd. Although Lombard et al. (2006) found a regional difference in environmental-sample culture results, no such difference was uncovered according to fecal culture or serum and milk ELISA. Certainly, more information is needed regarding the geographic distribution of bulkmilk MAP contamination. The findings of our study may provide the first clue regarding the geographical patterns of MAP-positive bulk-tank samples from US herds.

Although several groups have found that the effect of herd size on infection prevalence may be small and region-dependent (Collins et al., 1994) or negligible (van Schaik et al., 2003), there is much supporting evidence to corroborate an increased MAP prevalence among larger herds (e.g., Wells and Wagner, 2000; Crossley et al., 2005). Although no effect of herd size was uncovered in our linear model, farms with a herd size of $>200$ (compared with $<100$ ) had significantly higher odds in the logistic model of being PCR positive in the bulk tank. The lack of difference between small and medium-sized farms suggests a nonlinear relationship and the possibility of a threshold level to breach. Descriptively, large farms accounted for only $13.36 \%$ of farms in the study, but $33.34 \%$ of PCR positive farms were in this large herd-size category. Hypotheses for why a large herd size might be related to higher MAP levels are generally speculative. According to Crossley et al. (2005), the probability of having a subclinically infected cow in the herd is greater for larger farms, and the infection status of larger herds may be more difficult to monitor. Additionally, because larger herds may have increased stocking densities, MAP exposure based on relative contact could be heightened.

Wells and Wagner (2000) noted an association between the housing style for preweaned heifer calves and herd-level MAP prevalence. In the univariable analysis stage, we identified an association between fall housing of preweaned heifers in a multiple-animal pen and a PCR positive bulk-tank status. For spring housing, the difference was not as pronounced; however, a positive bulk-milk PCR was associated with farms on which the housing system implied calf-calf or cow-calf contact (i.e., multiple animal areas, or pasture/drylot) compared with farms practicing individual or restrictive housing (i.e., individual animal area, individual hutch, or tied in a barn).

Another variable found to be significant at the univariable stage was presence of scours in calves. Sorge et al. (2012) reported a lower incidence of calf scours among herds that tested negative for MAP antibodies (via serum ELISA) compared with test-positive herds. This finding is consistent with observations of van Roermund et al. (2007), who noted that calves, once infected, are able to horizontally transmit the infection to other calves in a relatively short period, likely by the fecal-oral route. In a study by Mortier et al. (2014), calves ( 1 yr of age and younger) experimentally inoculated with MAP showed a peak in MAP fecal shedding 2 mo after inoculation, with the first fecal positive at $0.5 \mathrm{mo}$, supporting the hypothesis of calf-to-calf transmission. The researchers posited that transmission potential between calves would understandably be heightened in a group-housing system. Although these types of relationships were not found to be significant in our multivariable logistic model, perhaps due to the necessary inclusion of several study design variables, they seem worth considering as potential risk factors for bulk milk contamination.

To fully capture the effect of environmental predictors on bulk-tank MAP status, more in-depth investigation of within-herd prevalence may be required. An advantage to a cross-sectional study such as this one is that it provides a snapshot of a variety of parameters from a large number of distinct farm environments; however, data of this type do not allow for investigation of within-herd dynamics over time.

Data obtained in a cross-sectional manner, without the benefit of repeated sampling, may be more susceptible to misclassification, particularly in low-prevalence herds (Lavers et al., 2013). Hence, longitudinal research on the association between within-herd prevalence, environmental load, bulk-milk ELISA, and bulk-milk PCR, is warranted. Lavers et al. (2013) longitudinally investigated the relationship between within-herd prevalence (determined by pooled fecal culture) and environmental culture results in a subset of Canadian herds; logically, the sensitivity of the environmental cultures increased alongside the level of within-herd fecal culture positives. Equivalent conclusions have been reached with respect to sensitivity of milk ELISA testing and fecal culture; specifically, as within-herd prevalence increases, so too does herd-level sensitivity for individual milk ELISA tests (McKenna et al., 2005; Lavers et al., 2014). How the relationship between bulk- 
tank PCR and ELISA results might differ based upon the within-farm prevalence and level of environmental burden has not yet been investigated. Additionally, it remains to be explored how the bulk-tank PCR-ELISA relationship could shift as herds alter management practices and change subtly in prevalence over time.

\section{CONCLUSIONS}

A substantial level of concordance between bulk-milk MAP ELISA and PCR in 286 samples was corroborated by fitting 2 regression models. Despite the agreement between high ELISA titers and positive PCR results in the bulk tank, the majority of high-ELISA farms were PCR negative, suggesting that such farms are largely able to maintain satisfactory hygiene and management despite an on-farm presence of MAP. Therefore, ELISA is not a perfect predictor of bulk-milk contamination with MAP pathogens themselves and is best used in combination with bulk-milk PCR to identify high-risk farms. An interaction may exist between production type and ELISA result on PCR results: organic farms demonstrated a higher probability of PCR-positive status with increasing ELISA titer compared with conventional farms. Additionally, a PCR-positive bulk tank was associated with large herd sizes and with a New York State location.

\section{ACKNOWLEDGMENTS}

The authors thank the USDA for funding (project USDA 2011-06385). We are grateful to Susan McAdams Gallagher and Ray Sweeney (University of Pennsylvania, Kennett Square) for assistance with PCR technique, provision of MAP bacteria, and continued support. We also thank Robab Katani, Lingling Li, and Vivek Kapur (Penn State University, University Park) for the K-10 MAP and associated protocols. Many thanks to Rebecca Craver (Cornell University, Ithaca, NY) for sharing her expertise with MAP and to members of the Schukken Laboratory at Cornell (Anja Sipka, Suzanne Klaessig, and Brianna Pomeroy) for advice.

\section{REFERENCES}

Alinovi, C. A., M. P. Ward, T. L. Lin, G. E. Moore, and C. C. Wu. 2009. Real-time PCR, compared to liquid and solid culture media and ELISA, for the detection of Mycobacterium avium ssp. paratuberculosis. Vet. Microbiol. 136:177-179.

Bosshard, C., R. Stephan, and T. Tasara. 2006. Application of an F57 sequence-based real-time PCR assay for Mycobacterium paratuberculosis detection in bulk tank raw milk and slaughtered healthy dairy cows. J. Food Prot. 69:1662-1667.

Cazer, C. L., R. M. Mitchell, K. M. Cicconi-Hogan, M. Gamroth, R. M. Richert, P. L. Ruegg, and Y. H. Schukken. 2013. Associations between Mycobacterium avium ssp. paratuberculosis antibodies in bulk tank milk, season of sampling and protocols for managing infected cows. BMC Vet. Res. 9:234-240.

Cetinkaya, B., K. Egan, D. A. Harbour, and K. L. Morgan. 1996. An abattoir-based study of the prevalence of subclinical Johne's disease in adult cattle in south west England. Epidemiol. Infect. 116:373-379.

Cicconi-Hogan, K. M., M. Gamroth, R. Richert, P. L. Ruegg, K. E. Stiglbauer, and Y. H. Schukken. 2013. Associations of risk factors with somatic cell count in bulk tank milk on organic and conventional dairy farms in the United States. J. Dairy Sci. 96:3689-3702.

Collins, M. T. 2011. Diagnosis of paratuberculosis. Vet. Clin. North Am. Food Anim. Pract. 27:581-591.

Collins, M. T., D. C. Sockett, W. J. Goodger, T. A. Conrad, C. B. Thomas, and D. J. Carr. 1994. Herd prevalence and geographic distribution of, and risk factors for, bovine paratuberculosis in Wisconsin. J. Am. Vet. Med. Assoc. 204:636-641.

Corti, S., and R. Stephan. 2002. Detection of Mycobacterium avium subspecies paratuberculosis specific IS900 insertion sequences in bulk-tank milk samples obtained from different regions throughout Switzerland. BMC Microbiol. 2:15-21.

Crossley, B. M., F. J. Zagmutt-Vergara, T. L. Fyock, R. H. Whitlock, and I. A. Gardner. 2005. Fecal shedding of Mycobacterium avium ssp. paratuberculosis by dairy cows. Vet. Microbiol. 107:257-263.

Doyle, T. M. 1956. Johne's disease. Vet. Rec. 68:869-878.

Eisenberg, S. W., R. Chuchaisangrat, M. Nielen, and A. Koets. 2013. Relationship between presence of cows with milk positive for Mycobacterium avium ssp. paratuberculosis-specific antibody by enzyme-linked immunosorbent assay and viable $M$. avium ssp. paratuberculosis in dust in cattle barns. Appl. Environ. Microbiol. 79:5458-5464.

Eltholth, M. M., V. R. Marsh, S. Van Winden, and F. J. Guitian. 2009. Contamination of food products with Mycobacterium avium paratuberculosis: A systematic review. J. Appl. Microbiol. 107:1061-1071.

Gao, A., L. Mutharia, M. Raymond, and J. Odumeru. 2007. Improved template DNA preparation procedure for detection of Mycobacterium avium ssp. paratuberculosis in milk by PCR. J. Microbiol. Methods 69:417-420.

Gao, A., J. Odumeru, M. Raymond, and L. Mutharia. 2005. Development of improved method for isolation of Mycobacterium avium ssp. paratuberculosis from bulk tank milk: Effect of age of milk centrifugation, and decontamination. Can. J. Vet. Res. 69:81-87.

Grant, I. R., H. J. Ball, and M. T. Rowe. 2002. Incidence of Mycobacterium paratuberculosis in bulk raw and commercially pasteurized cows' milk from approved dairy processing establishments in the United Kingdom. Appl. Environ. Microbiol. 68:2428-2435.

Grant, I. R., A. G. Williams, M. T. Rowe, and D. D. Muir. 2005. Efficacy of various pasteurization time-temperature conditions in combination with homogenization on inactivation of Mycobacterium avium ssp. paratuberculosis in milk. Appl. Environ. Microbiol. 71:2853-2861.

Herthnek, D., S. S. Nielsen, A. Lindberg, and G. Bölske. 2008. A robust method for bacterial lysis and DNA purification to be used with real-time PCR for detection of Mycobacterium avium ssp. paratuberculosis in milk. J. Microbiol. Methods 75:335-340.

Khol, J. L., M. Wassertheurer, E. Sodoma, S. Revilla-Fernández, J. Damoser, E. Österreicher, M. Dünser, U. Kleb, and W. Baumgartner. 2013. Long-term detection of Mycobacterium avium subspecies paratuberculosis in individual and bulk tank milk from a dairy herd with a low prevalence of Johne's disease. J. Dairy Sci. 96:35173524 .

Lavers, C. J., H. W. Barkema, I. R. Dohoo, S. L. B. McKenna, and G. P. Keefe. 2014. Evaluation of milk ELISA for detection of $M y$ cobacterium avium subspecies paratuberculosis in dairy herds and association with within-herd prevalence. J. Dairy Sci. 97:299-309.

Lavers, C. J., S. L. McKenna, I. R. Dohoo, H. W. Barkema, and G. P. Keefe. 2013. Evaluation of environmental fecal culture for $M y$ cobacterium avium subspecies paratuberculosis detection in dairy herds and association with apparent within-herd prevalence. Can. Vet. J. 54:1053-1060. 
Li, L., J. P. Bannantine, Q. Zhang, A. Amonsin, B. J. May, D. Alt, N. Banerji, S. Kanjilal, and V. Kapur. 2005. The complete genome sequence of Mycobacterium avium subspecies paratuberculosis. Proc. Natl. Acad. Sci. USA 102:12344-12349.

Lombard, J. E., I. A. Gardner, S. R. Jafarzadeh, C. P. Fossler, B. Harris, R. T. Capsel, B. A. Wagner, and W. O. Johnson. 2013. Herd-level prevalence of Mycobacterium avium subspecies paratuberculosis infection in United States dairy herds in 2007. Prev. Vet. Med. 108:234-238.

Lombard, J. E., B. A. Wagner, R. L. Smith, B. J. McCluskey, B. N. Harris, J. B. Payeur, F. B. Garry, and M. D. Salman. 2006. Evaluation of environmental sampling and culture to determine Mycobacterium avium subspecies paratuberculosis distribution and herd infection status on US dairy operations. J. Dairy Sci. 89:4163-4171.

Manning, E. J., and M. T. Collins. 2001. Mycobacterium avium ssp. paratuberculosis: Pathogen, pathogenesis and diagnosis. Rev. Sci. Tech. 20:133-150.

McKenna, S. L. B., G. P. Keefe, H. W. Barkema, and D. C. Sockett. 2005. Evaluation of three ELISAs for Mycobacterium avium ssp. paratuberculosis using tissue and fecal culture as comparison standards. Vet. Microbiol. 110:105-111.

Mortier, R. A., H. W. Barkema, K. Orsel, R. Wolf, and J. De Buck. 2014. Shedding patterns of dairy calves experimentally infected with Mycobacterium avium subspecies paratuberculosis. Vet. Res. 45:71-80.

Nielsen, S. S., S. M. Thamsborg, H. Houe, and V. Bitsch. 2000. Bulktank milk ELISA antibodies for estimating the prevalence of paratuberculosis in Danish dairy herds. Prev. Vet. Med. 44:1-7.

Odumeru, J., A. Gao, S. Chen, M. Raymond, and L. Mutharia. 2001. Use of the bead beater for preparation of Mycobacterium paratuberculosis template DNA in milk. Can. J. Vet. Res. 65:201-205.

Over, K., P. G. Crandall, C. A. O'Bryan, and S. C. Ricke. 2011. Current perspectives on Mycobacterium avium ssp. paratuberculosis, Johne's disease, and Crohn's disease: A review. Crit. Rev. Microbiol. 37:141-156.

Pickup, R. W., G. Rhodes, T. J. Bull, S. Arnott, K. Sidi-Boumedine, M. Hurley, and J. Hermon-Taylor. 2006. Mycobacterium avium ssp. paratuberculosis in lake catchments, in river water abstracted for domestic use, and in effluent from domestic sewage treatment works: Diverse opportunities for environmental cycling and human exposure. Appl. Environ. Microbiol. 72:4067-4077.

Richert, R. M., K. M. Cicconi, M. J. Gamroth, Y. H. Schukken, K. E. Stiglbauer, and P. L. Ruegg. 2013. Management factors associated with veterinary usage by organic and conventional dairy farms. J. Am. Vet. Med. Assoc. 242:1732-1743.

Ruzante, J. M., I. A. Gardner, J. S. Cullor, W. L. Smith, J. H. Kirk, and J. M. Adaska. 2008. Isolation of Mycobacterium avium ssp. paratuberculosis from waste milk delivered to California calf ranches. Foodborne Pathog. Dis. 5:681-686.

Schukken, Y. H., R. H. Whitlock, D. Wolfgang, Y. T. Grohn, A. Beaver, J. VanKessel, M. Zurakowski, and R. Mitchell. 2015. Longitudinal data collection of Mycobacterium avium subspecies paratuberculosis infections in dairy herds: The value of precise field data. Vet. Res. 46:65.

Singh, S. V., A. V. Singh, R. Singh, K. S. Sandhu, P. K. Singh, J. S. Sohal, V. K. Gupta, and V. S. Vihan. 2007. Evaluation of highly sensitive indigenous milk ELISA kit with fecal culture, milk culture and fecal-PCR for the diagnosis of bovine Johne's disease (BJD) in India. Comp. Immunol. Microbiol. Infect. Dis. 30:175-186.

Slana, I., P. Kralik, A. Kraklova, V. Babak, and I. Pavlik. 2012. Short communication: Examination of milk filters by real-time PCR as a herd-level indicator of the presence of Mycobacterium avium subspecies paratuberculosis in dairy herds. J. Dairy Sci. 95:1162-1165.
Slana, I., F. Paolicchi, B. Janstova, P. Navratilova, and I. Pavlik. 2008. Detection methods for Mycobacterium avium subsp. paratuberculosis in milk and milk products: A review. Vet. Med. Czech 53:283-306.

Sorge, U. S., K. Lissemore, A. Godkin, J. Jansen, S. Hendrick, S. Wells, and D. F. Kelton. 2012. Risk factors for herds to test positive for Mycobacterium avium ssp. paratuberculosis-antibodies with a commercial milk enzyme-linked immunosorbent assay (ELISA) in Ontario and western Canada. Can. Vet. J. 53:963-970.

Stiglbauer, K. E., K. M. Cicconi-Hogan, R. Richert, Y. H. Schukken, P. L. Ruegg, and M. Gamroth. 2013. Assessment of herd management on organic and conventional dairy farms in the United States. J. Dairy Sci. 96:1290-1300.

Streeter, R. N., G. F. Hoffsis, S. Bech-Nielsen, W. P. Shulaw, and D. M. Rings. 1995. Isolation of mycobacterium paratuberculosis from colostrum and milk of subclinically infected cows. Am. J. Vet. Res. $56: 1322-1324$.

Sung, N., and M. T. Collins. 1998. Thermal tolerance of Mycobacterium paratuberculosis. Appl. Environ. Microbiol. 64:999-1005.

Sweeney, R. W., R. H. Whitlock, and A. E. Rosenberger. 1992. Mycobacterium paratuberculosis cultured from milk and supramammary lymph nodes of infected asymptomatic cows. J. Clin. Microbiol. $30: 166-171$

van Roermund, H. J. W., D. Bakker, P. T. J. Willemsen, and M. C. M. De Jong. 2007. Horizontal transmission of Mycobacterium avium ssp. paratuberculosis in cattle in an experimental setting: Calves can transmit the infection to other calves. Vet. Microbiol. 122:270-279

van Schaik, G., Y. H. Schukken, C. Crainiceanu, J. Muskens, and J. A. VanLeeuwen. 2003. Prevalence estimates for paratuberculosis adjusted for test variability using Bayesian analysis. Prev. Vet. Med. 60:281-295.

Van Weering, H., G. Van Schaik, A. Van Der Meulen, M. Waal, P. Franken, and K. Van Maanen. 2007. Diagnostic performance of the Pourquier ELISA for detection of antibodies against Mycobacterium avium subspecies paratuberculosis in individual milk and bulk milk samples of dairy herds. Vet. Microbiol. 125:49-58.

Weber, M. F., M. Nielen, A. G. Velthuis, and H. J. van Roermund. 2008. Milk quality assurance for paratuberculosis: Simulation of within-herd infection dynamics and economics. Vet. Res. 39:12. http://dx.doi.org/10.1051/vetres:2007050.

Wells, S. J., and B. A. Wagner. 2000. Herd-level risk factors for infection with Mycobacterium paratuberculosis in US dairies and association between familiarity of the herd manager with the disease or prior diagnosis of the disease in that herd and use of preventive measures. J. Am. Vet. Med. Assoc. 216:1450-1457.

Whan, L. B., I. R. Grant, H. J. Ball, R. Scott, and M. T. Rowe. 2001. Bactericidal effect of chlorine on Mycobacterium paratuberculosis in drinking water. Lett. Appl. Microbiol. 33:227-231.

Whittington, R. J., I. B. Marsh, and L. A. Reddacliff. 2005. Survival of Mycobacterium avium ssp. paratuberculosis in dam water and sediment. Appl. Environ. Microbiol. 71:5304-5308.

Wilson, D. J., K. Rood, P. Biswas, and T. M. Byrem. 2010. Herd-level prevalence of Johne's disease in Utah and adjacent areas of the intermountain west as detected by a bulk-tank milk surveillance project. J. Dairy Sci. 93:5792-5797.

Wynne, J. W., T. Seemann, D. M. Bulach, S. A. Coutts, A. M. Talaat, and W. P. Michalski. 2010. Resequencing the Mycobacterium avium ssp. paratuberculosis K10 genome: Improved annotation and revised genome sequence. J. Bacteriol. 192:6319-6320.

Zwald, A. G., P. L. Ruegg, J. B. Kaneene, L. D. Warnick, S. J. Wells, C. Fossler, and L. W. Halbert. 2004. Management practices and reported antimicrobial usage on conventional and organic dairy farms. J. Dairy Sci. 87:191-201. 